\title{
Gauging Clinical Practice: Surgical Navigation for Total Hip Replacement
}

\author{
James E. Moody Jr. ${ }^{1}$; Anthony M. DiGioia, MD ${ }^{1,2}$; Branislav Jaramaz ${ }^{1,2}$; \\ Mike Blackwell ${ }^{2}$; Bruce Colgan ${ }^{1}$; Constantinos Nikou ${ }^{2}$
}

${ }^{1}$ Center for Orthopaedic Research, Shadyside Medical Center, 5200 Centre Avenue, Suite 309, Pittsburgh, PA 15232, TEL (412) 623-2673, FAX (412) 623-1108

\{moody, branko, colgan\} ecor.ssh.edu

${ }^{2}$ Center for Medical Robotics and Computer Assisted Surgery, Robotics Institute, Carnegie Mellon University, Pittsburgh, PA 15213

\{digioia, mkb, costa\}@cmu.edu

\begin{abstract}
HipNav is a hip implant navigation and guidance system which helps surgeons plan acetabular implant orientation preoperatively, and guides them intraoperatively to achieve this intended plan. Through this process, these technologies are used to measure, gauge, and quantify current clinical practice. With HipNav, surgeons plan and execute the procedure in the 3-D realm with accurate patient models, taking advantage of anatomic information normally lost through traditional planning methods. During surgery HipNav tracks the alignment tool with respect to the pelvis, compensating for any pelvic motion, thereby ensuring an accurate measurement of alignment and placement according to the plan.

This past year has seen the system move out of the developmental laboratory setting and into the operating room environment. In addition to its primary clinical function as a surgical assist device, through its measurement and tracking capabilities HipNav has likewise emerged as a powerful research tool. With HipNav we are now able to measure, for example, how the pelvis moves during surgery, how cup placement shifts during the press fit process, and how well traditional implant guides and strategies compare with new strategies based on more complete patient-specific models.

HipNav is still an emerging technology and a continually evolving system. However, data collected to date through the HipNav project have provided new insight into aspects of the total hip replacement process, challenging commonly held assumptions concerning acetabular alignment. Most importantly, HipNav has indications for some areas of established practice that may benefit from reexamination.
\end{abstract}

\section{Introduction}

Although many factors may contribute to dislocation following total hip replacement (THR) surgery, one of the most common negative influences is the malposition of the acetabular portion of the implant [3], [4]. A non-optimal placement can lead to impingement between the rim of the acetabular cup and the neck of the femoral component. This impingement acts as a fulcrum, such that any attempted motion beyond this point will result in subluxation or even dislocation. In less severe cases malposition can accelerate the wear of the polyethylene liner, generating debris which, in turn, can contribute to early implant loosening or failure[8], [12]. 
Clearly, the best strategy is to place the acetabular component to minimize or completely avoid the potential for impingement during routine activities of daily living [2], [7]. However, current methods do not provide clinicians with adequate tools to develop optimal implant placement strategies, nor do they provide the means by which the desired plan can be reliably implemented.

The traditional planning method of using a single anterior-posterior $\mathrm{X}$-ray with a transparent template overlay is inherently inaccurate for alignment in that much 3-D information is eliminated during the projection to a 2-D radiograph. Also, this technique does not take into account functional pelvic positions unique to the patient. For instance, pelvic pitch varies significantly as a person moves from a standing to a sitting position; the range of this pitch can differ significantly between patients. These are factors that should be considered in the operative plan.

Current practice of acetabular implant placement relies on a rigid mechanical guide attached to the component insertion tool. This guide may work satisfactorily if the surgeon can ascertain that the pelvis is precisely and securely placed in the known orientation for which the guide was designed. However, precise and repeatable pelvic alignment in an operating room environment is not easily achieved, if even possible, without extensive external fixation. A mechanical implant guide does not account for any deviation in pelvic position, nor does it correct for any peculiarities resulting from the patient's unique anatomical structure or natural posture [6].

Until now there has not been a mechanism by which the implant tool could be accurately oriented to the pelvis. To this end we have developed HipNav, a system coupling a preoperative planner with an intraoperative navigation system. Our approach uses accurate 3-D patient pelvic models to assist surgeons in determining and achieving the optimal acetabular implant orientation, regardless of pelvic motion and orientation on the operating room table.

Inherent in the HipNav system are powerful tracking and measurement utilities. Aside from its intended clinical use we can take advantage of these capabilities to measure and quantify various aspects of the THR procedure.

\section{System}

Previous papers have described components of the HipNav system and its validation in great detail [1], [11]. However, a brief system overview and examination of our methodology is necessary to provide an adequate insight to our approach and findings.

\subsection{HipNav Components}

HipNav is comprised of three functional components:

- A preoperative planner.

- A range-of-motion simulator.

- An intraoperative navigation and guidance system.

The preoperative planner presents a 3-D surface model of the patient's pelvis to the surgeon, who can then specify the implant size and location. Figure 1 shows an 
example of the implant placement screen in which the acetabular implant is modeled as a sphere, thus the orthagonal cross sections appear as circles. The surface model in this particular example depicts only the right half of a pelvis. The centroid of the implant appears as a small dot in the center of the acetabulum. For any given point in the pelvis, simulated sagittal and coronal CT views are presented along with the traditional transverse CT view. These three orthogonal views follow the center of the implant spheroid: as the implant is moved the views adjust accordingly. Thus, the surgeon can fine-tune implant placement along all three axes.

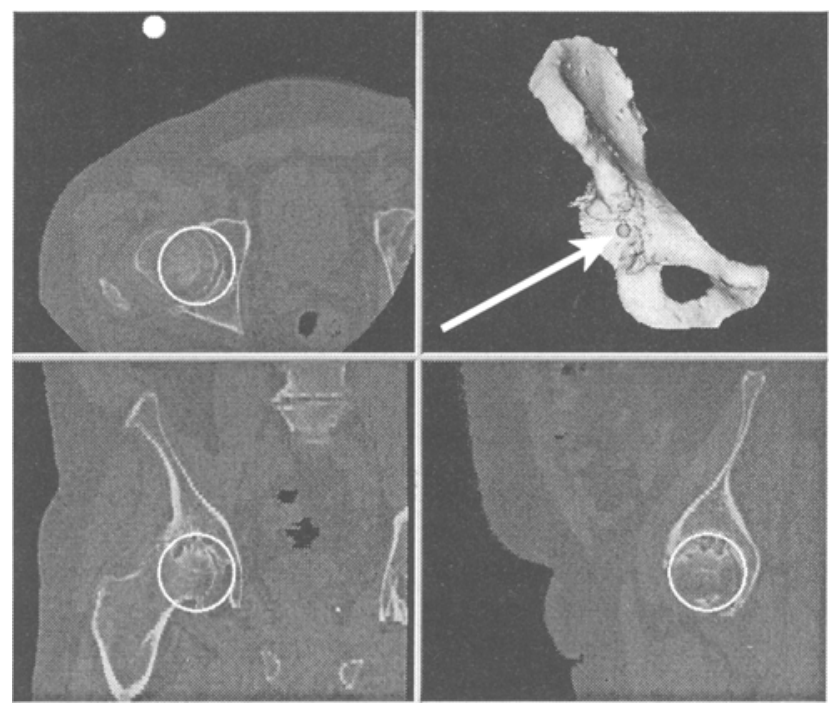

Figure 1: The implant placement screen of the HipNav planner. The arrow indicates the centroid of the spherical implant model.

Once the size and location of the implant are determined, the implant can then be oriented through the orientation utility. Coupled with this utility is the second functional component of HipNav, the range-of-motion simulator. The simulator dynamically predicts impingement for various leg motions given the size, geometry, and orientation of the implant components selected. Thus, the surgeon can optimize the plan (adding more version, for example) to adjust away from an orientation which might otherwise be predisposed to impingement.

A portion of the orientation utility screen is presented in Figure 2, showing both a pelvis with simulated implant in a test orientation, and the resulting predicted functional range of motion.

The third component of HipNav is the intraoperative navigation and guidance system (IOS). Optical tracking hardware monitors the locations and orientations of the data point collection probe and the implant insertion tool. Early in the surgical procedure an optical tracking target is rigidly attached to the pelvis, and after the calibration and registration processes all components can be located and tracked within the operating field accurately and in real time. IOS couples this tracking capability with the 


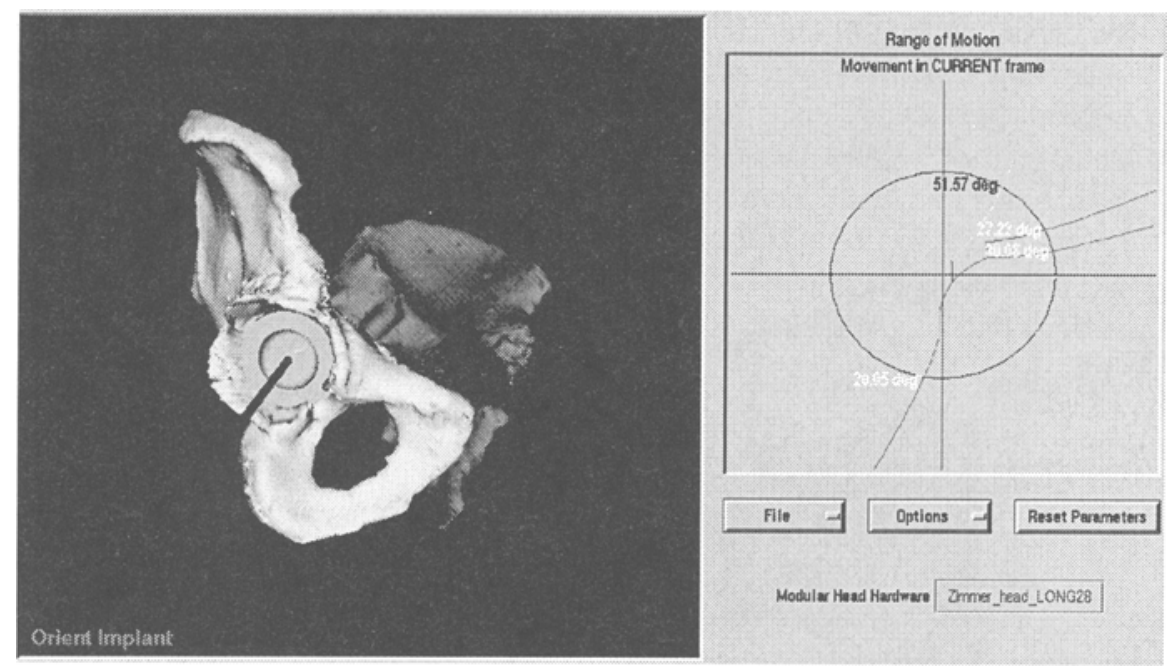

Figure 2: A sample implant orientation and resulting range-of-motion simulation from the HipNav planner.

patient's own preoperative plan and presents this information to the surgeon via various context relevant displays throughout the entire procedure.

\subsection{Data Preparation}

In preparation for a HipNav case, lateral standing and sitting $\mathrm{X}$-rays of the pelvis are taken in addition to the standard anterior-posterior view. From this lateral series the surgeon can estimate a functional range of pelvic motion, which usually has an impact on the implant strategy.

A CT scan of the pelvis is also performed, from which 2-D contours are extracted, and ultimately a 3-D surface model is constructed. Examples of these models were shown in Figures 1 and 2. The models are also used by the intraoperative system.

\subsection{Definition of the Anterior Pelvic Plane}

Given the wide variation in pelvic structure and disposition between patients it is imperative to have a simple but robust definition for orientation against which rotations and translations can be described. HipNav measurements are based on what we call the "anterior pelvic plane" (a concept independently proposed by Lewinnek [5]). Assuming a symmetric pelvis, the anterior pelvic plane is simply the plane defined by the most anterior points on the pubic tubercles and on the anterior superior iliac spines. To illustrate, if a pelvic model were held against a window it would touch at these points, and the plane defined by the window would be coincident with the anterior pelvic plane. Conceptually simple, the anterior pelvic plane has these advantages:

- It is intuitive, as it is nearly vertical in a standing person.

- Since it is anatomically based it can be easily measured by physical exam.

- It can be precisley measured in X-rays.

- It is easily determined in 3-D reconstructions. 
Conversely, implant manufacturer guidlines typically refer to a pelvic coronal plane, the definition and determination of which is somewhat more subjective.

\subsection{Data Collection Devices}

HipNav's tracking system ${ }^{1}$, capable of sub-millimeter accuracy, can measure the exact position and orientation of optical targets in the operating room. When these targets are attached to various tools, they then become position measuring devices in addition to their primary purpose. Data from these measurement devices can be collected automatically by the system, by the surgeon via a foot pedal, or by the system engineer at the console. All data are logged for intraoperative use as well as for postoperative analysis.

HipNav makes use of three such measurement devices: a point probe, an implant insertion tool, and the patient's own pelvis.

The Point Probe. The point probe (a.k.a, the data probe, or wand) is a specialized optical target with an integral stainless steel probe tip. Point data collection is achieved by the surgeon placing the tip of the data probe on a desired spot and striking a foot pedal to record the position. Point data contains no orientation information, just location coordinate measurements.

The Pelvis as a Measurement Tool. Once equipped with an optical target the pelvis becomes trackable both in location and in orientation. The target is rigidly fixed, therefore any motion in the pelvis results in target motion. The registration process (as described in the Methodology section) is performed so that the target/pelvic motion can be related to the computer's internal model.

A Trackable Implant Tool. The trackable alignment implant tool is simply the appropriate manufacturer's standard implant tool and mechanical guide to which an optical target has been securely affixed. Although its intended function is to set the alignment during press fit we use this now-trackable tool to collect abduction/flexion measurements in three different situations:

- We can measure alignment strategies before press fit.

- We can track the actual change in cup orientation during the press fit process.

- We can measure any effects of post-insertion steps. For example, we can detect any change due to the insertion of fixating screws, or any change resulting from the seating of the acetabular liner.

\section{Methodology}

The previous section introduced the measurement tools and described the types of measurements each can take. This section focuses on the different classes of measurements (gathered from any combination of the above devices), how they are measured, what the data show, and what conclusions (if any) can be inferred from the data.

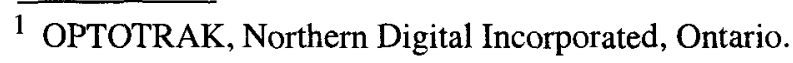




\subsection{Registration}

Using the data probe the surgeon is prompted to collect a series of points from designated locations on the surface of the pelvis, in effect creating a "point cloud" in computer memory. Since the pelvic model and the cloud of points are both based on the same physical pelvis, these two representations can be permuted until a precise surface match is found. Once this registration process is complete the conversion from the pelvic model to the operating room is well understood so that any measurements taken in the OR can be accurately mapped onto the computer model [9], [10].

\subsection{Pelvic Position Tracking}

Natural Pelvic Orientation vs. the Theoretical Neutral. The patient is positioned on the OR table in an attitude typical for a posterolateral approach: The patient is stabilized with a series of anterior and posterior blocks and a bean bag. The non-operative leg is secured to the table in approximately 45 degrees of flexion. The pelvis is now in the baseline position, and once the optical target is attached this position is recorded as the baseline neutral position. In the theoretical (or "ideal") neutral position assumed by mechanical guides the anterior pelvic plane would be perpendicular to the surface of the OR table, and parallel with its long axis.

Table 1 shows the deviation from theoretical neutral of the baseline (or natural) neutral position for each of the HipNav patients. Roll is defined as a rotation about the superior-inferior axis, pitch as a rotation about the lateral axis, and yaw as a rotation about the anterior-posterior axis of the pelvis.

We do not draw any direct conclusions from these data except that they demonstrate a wide variation in natural neutral positions. The best use of such data is in evaluating assumptions held in current practice.

For example, if it could be determined that current planning techniques are valid provided the actual pelvis is within $\pm 5^{\circ}$ on all axes of the theoretical neutral alignment, then only three of the 15 cases in Table 1 for which data are available would be in the tolerable range. This is, of course, a hypothetical example contrived just to show how such data might be used. It also makes the naive assumption that the same neutral postition can be maintained throughout the surgical procedure.

Pelvic Motion. HipNav monitors pelvic position throughout the surgery. In our current protocol a starting, or baseline, position is measured as soon as the optical target has been attached. Pelvic position measurements are subsequently taken after any large force has been applied to the pelvis. We typically take measurements after the femoral head has been removed, after reaming and after press fit, and we take an additional series during the final prosthetic range of motion tests.

The most intuitive representation of the motion data is an animated sequence. Figure 3 shows a sampling of frames from such a sequence for a representative HipNav case. In this particular example only the right half of the pelvis was actually reconstructed from CT data. In the interest of clarity this hemi-pelvis was reflected to produce a realistic-looking pelvic image.

The white outline represents the theoretical neutral orientation.

Figures $3 \mathrm{a}, 3 \mathrm{~b}$, and $3 \mathrm{c}$ (the left column) represent the pelvis in "relaxed" positions, i.e., where no force is being applied so the torso can return to a neutral state. Figures 
Table 1. Natural Pelvic Orientation - Deviation from Theoretical Neutral

\begin{tabular}{|c|c|c|c|}
\hline CASE \# & ROLL & PITCH & YAW \\
\hline \hline 1 & 17.4 & -4.0 & -2.3 \\
2 & $\mathrm{n} / \mathrm{a}$ & $\mathrm{n} / \mathrm{a}$ & $\mathrm{n} / \mathrm{a}$ \\
3 & -4.2 & 1.9 & 0.5 \\
4 & $\mathrm{n} / \mathrm{a}$ & $\mathrm{n} / \mathrm{a}$ & $\mathrm{n} / \mathrm{a}$ \\
5 & 21.4 & -13.7 & 5.7 \\
\hline 6 & $\mathrm{n} / \mathrm{a}$ & $\mathrm{n} / \mathrm{a}$ & $\mathrm{n} / \mathrm{a}$ \\
7 & -9.6 & -6.6 & 6.5 \\
8 & 18.5 & 4.6 & 5.4 \\
9 & 5.7 & 0.9 & -1.1 \\
10 & -6.4 & -1.7 & -4.4 \\
\hline 11 & 2.0 & 3.7 & 1.5 \\
12 & 3.4 & -0.9 & -1.1 \\
13 & 5.1 & -1.6 & -1.4 \\
14 & -7.8 & 2.2 & -6.5 \\
15 & 10.8 & 3.8 & 9.1 \\
\hline 16 & 3.3 & 9.2 & 7.5 \\
17 & 4.5 & 14.8 & -3.3 \\
18 & -4.8 & 2.7 & -11.1 \\
\hline
\end{tabular}

$3 \mathrm{~d}, 3 \mathrm{e}$, and $3 \mathrm{f}$ (the right column) show examples of pelvic motion during the prosthetic range of motion tests in which some force is being applied to the leg.

These images illustrate very convincingly that although the patient is secured and stabilized the pelvis moves significantly. Even more provoking is the realization that when forces are removed the pelvis often does not return to the same neutral position. This demonstrates that the concept of a single neutral position is not realistic.

\subsection{Measurement of Placement Strategies}

The implant tool is used to measure cup abduction and flexion once the reaming process has been completed. The alignment implant tool is oriented according to some strategy, whether it is the manufacturer's mechanical guide according to the manufacturer's directions, the surgeon's preference based on his or her own experience and knowledge of anatomical cues, or even a new technique as described in a research paper. Once the tool is aligned according to a given scheme the true resulting abduction and flexion can be measured. Table 2 lists the measurements from the first 10 HipNav cases for two strategies (relying on the mechanical guide according to the manufacturer's guidelines, and relying on the surgeon's own experience and anatomical cues). The "HipNav" column contains the planned orientations, and in most cases this is what the surgeon chose to attempt for the final placement.

It is interesting to note that in all cases even though the same surgeon both planned the case and performed the operation there were sometimes significant differences in the preoperative plan and the surgeon's intraoperative strategy. Part of this is due to the limited anatomical cues visible at the incision site, especially with heavier patients. 
Neutral/resting positions.

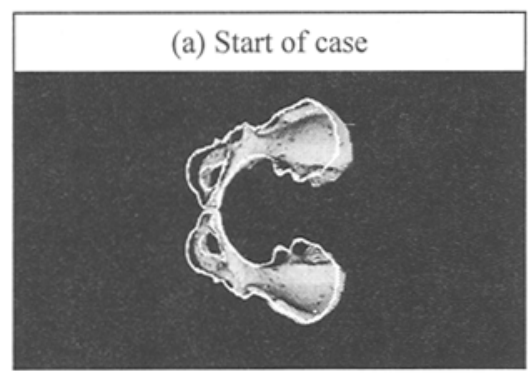

(b) After press fit

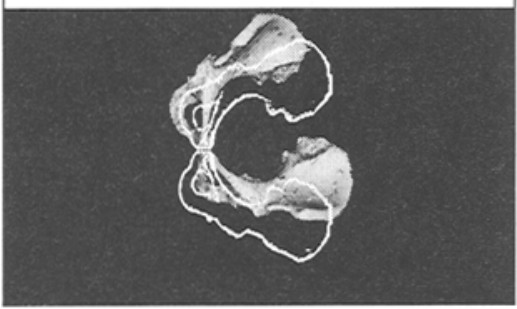

(c) After femoral component

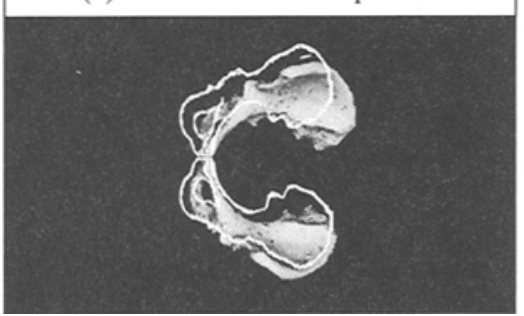

Positions during various ROM tests.

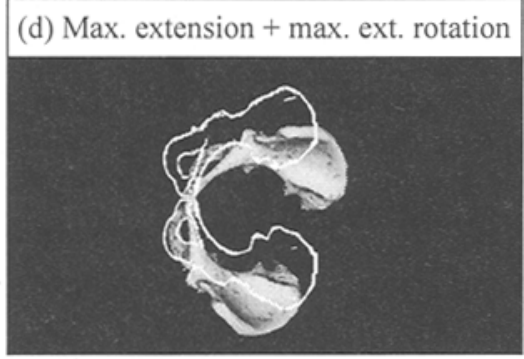

(e) Flexion $90^{\circ}+\max$. internal rotation

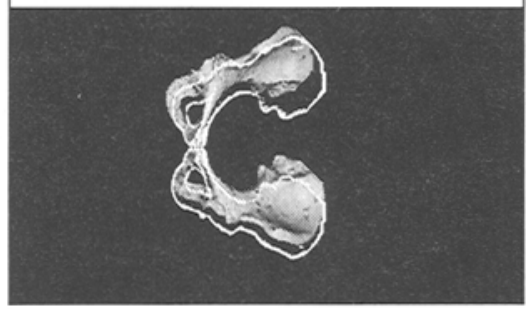

(f) Maximal flexion

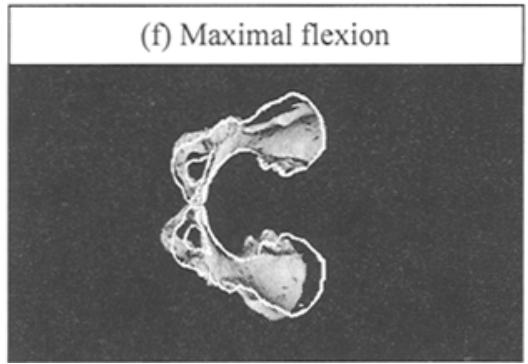

Figure 3: Representative snapshots of pelvic motion during a typical THR. The white outline indicates the ideal orientation as required by mechanical alignment guides.

\subsection{Cup Motion}

The last column in Table 2 lists the measured orientation of the actual implant placement. Compare this to the planned orientation in the previous column.

This shows that one of the most difficult tasks in the procedure is keeping the implant on an accurate course during press fit, even with the benefit of computer guidance. The implant is typically two to four millimeters larger than the prepared site. But because of tight fit and variances in bone density and quality the cup has a tendency to bind and torque during press fit.

Also, with a slightly rounded striking head on the pressfit tool it is difficult for surgeons to strike directly along the axis. Any off-center impact generates rotational forces about the moment arm defined by the insertion tool, contributing to cup rotation.

We are currently studying this phenomenon in greater detail, expecting that it will 
Table 2. Acetabular Implant Strategies; Final Position.(Measurements listed as abduction/anteversion)

\begin{tabular}{|c|c|c|c||c|}
\hline CASE \# & GUIDE & SURGEON & HIPNAV & FINAL \\
\hline \hline 1 & $40 /-9$ & $50 / 16$ & $48 / 19$ & $48 / 26$ \\
2 & $42 /-2$ & $48 / 24$ & $54 / 20$ & $51 / 27$ \\
3 & $41 / 9$ & $54 / 26$ & $54 / 20$ & $61 / 24$ \\
4 & n/a & $56 / 23$ & $53 / 16$ & $54 / 22$ \\
5 & $48 /-7$ & $48 / 16$ & $55 / 21$ & $52 / 16$ \\
\hline 6 & $43 / 19$ & $54 / 37$ & $56 / 23$ & $58 / 33$ \\
7 & $36 / 1$ & $45 / 32$ & $56 / 23$ & $47 / 20$ \\
8 & $44 / 3$ & $51 / 21$ & $54 / 20$ & $48 / 20$ \\
9 & $44 / 20$ & $54 / 33$ & $56 / 23$ & $53 / 16$ \\
10 & $44 / 5$ & $54 / 28$ & $54 / 20$ & $52 / 27$ \\
\hline
\end{tabular}

lead to better implant tool designs and techniques. With HipNav technology we can test the effectiveness of any improvements.

Cup motion can also result from post- press fit procedures. The implant insertion tool can be fitted with an adapter which fits snugly into the acetabular component liner (hooded or standard). From this the final abduction and flexion measurements are recorded. We use this to measure any cup orientation change due to the optional insertion of fixating screws, or even from the force of seating the liner itself.

\section{Conclusions}

We acknowledge that the subject pool is still too small to yield statistically rigorous clinical conclusions; likewise, the time period has been too brief to attempt any meaningful outcomes analysis. Even so, HipNav has convincingly demonstrated that commonly held assumptions regarding acetabular implant practice should be re-examined.

We have also shown that as a research tool HipNav provides many measurement utilities enabling investigation into varied aspects of total hip replacement:

- HipNav can be used to evaluate new tool designs. Our current data suggest that press fit tools that can deliver forces focused along the main axis may result in better cup placement. HipNav can be used to assess the effectiveness of the tool by tracking the press fit progress and measuring the final orientation.

- HipNav can be used to develop and evaluate new alignment strategies. We expect that new strategies will emerge, either based on new combinations of anatomical landmarks, or hybid approaches employing mechanical guides with anatomical landmarks. HipNav can be used to measure how these strategies perform in actual practice.

- HipNav can be used to develop accuracy requirements. HipNav can predict how deviations from alignment strategy assumptions affect functional range of motion, thereby helping to establish guidelines for safe practice.

- Intraoperatively, HipNav can be used to measure final implant placement, and can predict potential problems while they can be corrected. 
- HipNav research provides a means to disseminate new strategies and information to a wider surgical audience.

\section{$5 \quad$ References}

1. Blackwell M, Simon D, Rao S, DiGioia A: Technical report. Design and evaluation of 3-D pre-operative planning software: application to acetabular implant alignment. Carnegie Mellon University, 1996.

2. Daly PJ, Morrey BF: Operative correction of an unstable total hip arthroplasty. J Bone Joint Surg 74A: 1334-1343, 1992.

3. Hedlundh U, Sanzen L, Fredin H: The prognosis and treatment of dislocated total hip arthroplasties with a $22 \mathrm{~mm}$ head. J Bone Joint Surg 79B: 374-378, 1997.

4. Khan MAA, Brakenbury PH, Reynolds ISR: Dislocation following total hip replacement. J Bone Joint Surg 63B: 214-218, 1981.

5. Lewinnek GE, Lewis JL, Tarr R, et al.: Dislocations after total hip replacement arthroplasties. J Bone Joint Surg 60A: 217-220, 1978.

6. McCollum DE, Gray WJ: Dislocation after total hip arthroplasty: Causes and prevention. Clin Orthop 261: 159-170, 1990.

7. Ohlin A, Balkfors B: Stability of cemented sockets after 3-14 years. J Arthroplasty 7: 87-92, 1992.

8. Robinson RP, Simonian PT, Gradisar IM, Ching RP: Joint motion and surface contact area related to component position in total hip arthroplasty. J Bone Joint Surg 79B: 140-46, 1997.

9. Simon D, Hebert M, Kanade T: Techniques for fast and accurate intrasurgical registration. J Image Guid Surg 1: 17-29, 1995.

10. Simon DA: Fast and accurate shape-based registration. Technical Report, \#CMU-RI-TR-96-45. Carnegie Mellon University, 1996.

11. Simon DA, Jaramaz B, Blackwell M, et al.: Development and validation of a navigational guidance system for acetabular implant placement. First Joint Conference of CVRMed and MRCAS. Grenoble, France, Springer 583-592, 1997.

12. Yamaguchi M, Bauer T, Hashimoto Y: Three-dimensional analysis of multiple wear vectors in retrieved acetabular cups. J Bone Joint Surg 79A: 1539-1544, 1997. 